\title{
Partitioning Harary graphs into connected subgraphs containing prescribed vertices
}

\author{
Olivier Baudon Julien Bensmail Éric Sopena $\left.\right|^{k}$
}

Univ. Bordeaux, LaBRI, UMR 5800, F-33400 Talence, France

CNRS, LaBRI, UMR 5800, F-33400 Talence, France

received $24^{\text {th }}$ May 2012, revised $9^{\text {th }}$ Aug. 2013, $3^{\text {rd }}$ Dec. 2014, $4^{\text {th }}$ Dec. 2014, accepted $5^{\text {th }}$ Dec. 2014.

A graph $G$ is arbitrarily partitionable (AP for short) if for every partition $\left(n_{1}, n_{2}, \ldots, n_{p}\right)$ of $|V(G)|$ there exists a partition $\left(V_{1}, V_{2}, \ldots, V_{p}\right)$ of $V(G)$ such that each $V_{i}$ induces a connected subgraph of $G$ with order $n_{i}$. If, additionally, $k$ of these subgraphs $(k \leq p)$ each contains an arbitrary vertex of $G$ prescribed beforehand, then $G$ is arbitrarily partitionable under $k$ prescriptions (AP $+k$ for short). Every $\mathrm{AP}+k$ graph on $n$ vertices is $(k+1)$-connected, and thus has at least $\left\lceil\frac{n(k+1)}{2}\right\rceil$ edges. We show that there exist $\mathrm{AP}+k$ graphs on $n$ vertices and $\left\lceil\frac{n(k+1)}{2}\right\rceil$ edges for every $k \geq 1$ and $n \geq k$.

Keywords: arbitrarily partitionable graph, partition under prescriptions, Harary graph

\section{Introduction}

We denote by $V(G)$ and $E(G)$ the sets of vertices and edges, respectively, of a graph $G$. The order (resp. size) of $G$ is the cardinality of the set $V(G)$ (resp. $E(G)$ ). If $X$ is a subset of $V(G)$, then $G[X]$ denotes the subgraph of $G$ induced by $X$.

In the late 1970s, the following well-known result was proved.

Theorem 1 (Győri [5] and Lovász [7], independently). If $G$ is a k-connected graph, then, given a sequence $\left(v_{1}, v_{2}, \ldots, v_{k}\right)$ of $k$ distinct vertices of $G$ and a sequence $\left(n_{1}, n_{2}, \ldots, n_{k}\right)$ of $k$ positive integers adding up to $|V(G)|$, there exists a partition $\left(V_{1}, V_{2}, \ldots, V_{k}\right)$ of $V(G)$ such that $v_{i} \in V_{i}$, the subgraph $G\left[V_{i}\right]$ is connected, and $\left|V_{i}\right|=n_{i}$ for every $i \in\{1,2, \ldots, k\}$.

In this paper, we consider a more general partition problem resulting from the combination of the notion of arbitrarily partitionable graphs [1] with the constraint of prescribing a set of vertices from Theorem 1 . Let $G$ be a connected graph of order $n$. A sequence $\tau=\left(n_{1}, n_{2}, \ldots, n_{p}\right)$ of positive integers is admissible for $G$ if it performs a partition of $n$, that is if $\sum_{i=1}^{p} n_{i}=n$. If, additionally, we can partition $V(G)$ into $p$ parts $\left(V_{1}, V_{2}, \ldots, V_{p}\right)$ such that each $V_{i}$ induces a connected subgraph of $G$ with order $n_{i}$, then $\tau$ is realizable in $G$, the partition $\left(V_{1}, V_{2}, \ldots, V_{p}\right)$ being a realization of $\tau$ in $G$. If every admissible sequence

*Corresponding author, sopena@labri.fr

1365-8050 (C) 2014 Discrete Mathematics and Theoretical Computer Science (DMTCS), Nancy, France 
for $G$ is also realizable in $G$, then $G$ is arbitrarily partitionable (AP for short). The interested reader is referred to [1, 2, 6, 8] for a review of some results on AP graphs.

Now suppose that we still want to partition $G$ into an arbitrary number, say $p$, of connected subgraphs $G_{1}, G_{2}, \ldots, G_{p}$ of prescribed orders, but in such a way that for each $i \in\{1,2, \ldots, k\}$ with fixed $k \in\{1,2, \ldots, p\}$, the subgraph $G_{i}$ contains a vertex $v_{i}$ of $G$ arbitrarily chosen beforehand. To model this additional requirement, the definition of AP graphs can be strenghtened as follows [3]. A $k$-prescription of $G$ is a $k$-tuple $P=\left(v_{1}, v_{2}, \ldots, v_{k}\right)$ of $k$ distinct vertices of $G$. We say that a sequence $\tau=\left(n_{1}, n_{2}, \ldots, n_{p}\right)$ with $p \geq k$ elements is realizable in $G$ under $P$ if there exists a realization $\left(V_{1}, V_{2}, \ldots, V_{p}\right)$ of $\tau$ in $G$ such that the vertex $v_{i}$ belongs to $V_{i}$ for every $i \in\{1,2, \ldots, k\}$. Notice that we have adopted the convention that the elements of $\tau$ associated with the prescribed vertices are the first elements of $\tau$. We say that $G$ is $(p, k)$-partitionable if every sequence admissible for $G$ consisting of exactly $p$ elements is realizable in $G$ under every $k$-prescription. Finally, the graph $G$ is arbitrarily partitionable under $k$ prescriptions (AP+ $k$ for short) if $G$ is $(p, k)$-partitionable for every $p \in\{k, k+1, \ldots, n\}$.

According to these definitions, an AP+0 graph is an AP graph. Stated differently, Theorem 1 asserts that every $k$-connected graph is $(k, k)$-partitionable. In the same flavour, note that every $k$-connected graph with $k \geq 2$ is trivially $(k, k-1)$-partitionable. Hence, when dealing with a $k$-connected graph, we only consider sequences with strictly more than $k$ elements throughout this paper. It also has to be known that deciding whether a sequence is realizable in a graph under a prescription is NP-complete in general, even when the sequence or the prescription has a fixed number of elements [4].

Only a few classes of AP+ $k$ graphs are known. For every $k \geq 1$, the set of complete graphs on at least $k$ vertices is a trivial class of $\mathrm{AP}+k$ graphs, these graphs having the largest possible size. Regarding graphs with less edges, it was proved in [3] that $k^{t h}$ powers of paths (resp. cycles) are $\mathrm{AP}+(k-1)$ (resp. $\mathrm{AP}+(2 k-1)$ ) for every $k \geq 1$, these results being tight (i.e. we cannot always partition these graphs when more prescriptions are requested).

In this work, we investigate the least possible size of an $\mathrm{AP}+k$ graph. In this scope, we focus on optimal $\mathrm{AP}+k$ graphs, i.e. on $\mathrm{AP}+k$ graphs with the least possible number of edges regarding their order and connectivity. This is done by studying the family of well-known Harary graphs. After having introduced some notation and preliminary results in Section 2, we prove some more results regarding the partition of powers of paths or cycles in Section 3 These results are then used to show, in Section 4 , that every $(k+1)$-connected Harary graph is an optimal $\mathrm{AP}+k$ graph for every $k \neq 2$. We finally deal with 3 connected Harary graphs in Section 5 In particular, we show that these graphs are not necessarily AP+2. We however provide another class of optimal AP+2 graphs instead. All these results imply that, for every $k \geq 1$ and $n \geq k$, every optimal AP+k graph with order $n$ has size $\left\lceil\frac{n(k+1)}{2}\right\rceil$.

\section{Definitions, notation, and preliminary results}

A subgraph $H$ of a graph $G$ is a spanning subgraph of $G$ if $V(H)=V(G)$. We also say that $G$ is spanned by $H$. Given an integer $k \geq 1$, the $k^{\text {th }}$ power of $G$, denoted by $G^{k}$, is the graph with the same vertex set as $G$, two vertices of $G^{k}$ being adjacent if they are at distance at most $k$ in $G$. We denote by $P_{n}$ (resp. $C_{n}$ ) the path (resp. cycle) on $n$ vertices. The vertices of $P_{n}$ or $C_{n}$ are consecutively denoted by $v_{0}, v_{1}, \ldots, v_{n-1}$. Regarding $P_{n}$, the vertices $v_{0}$ and $v_{n-1}$ are its first and last vertices, respectively, sometimes called its endvertices. We use the same terminology to deal with the vertices of $P_{n}^{k}\left(\right.$ resp. $\left.C_{n}^{k}\right)$ according to its natural spanning $P_{n}$ (resp. $C_{n}$ ). 


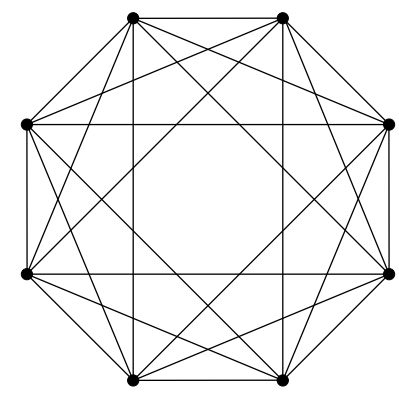

(a) $H_{6,8}$.

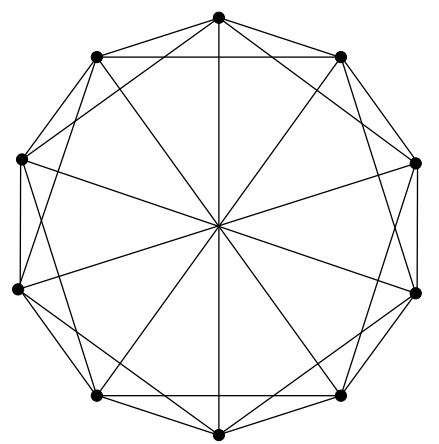

(b) $H_{5,10}$.

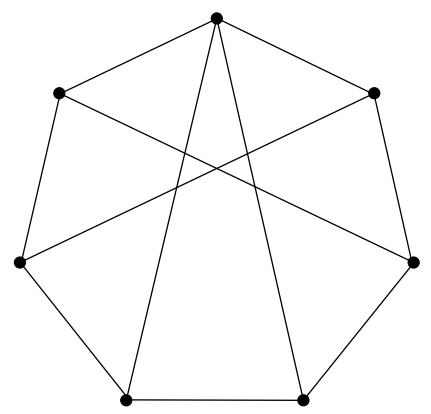

(c) $H_{3,7}$.

Fig. 1: Three examples of Harary graphs.

Let $k \geq 1$ and $n \geq k$ be two integers. The $k$-connected Harary graph on $n$ vertices, denoted by $H_{k, n}$, has a vertex set $\left\{v_{0}, v_{1}, \ldots, v_{n-1}\right\}$ and the following edges:

- if $k=2 r$ is even, then two vertices $v_{i}$ and $v_{j}$ are linked if and only if $i-r \leq j \leq i+r$;

- if $k=2 r+1$ is odd and $n$ is even, then $H_{k, n}$ is obtained by joining $v_{i}$ and $v_{i+\frac{n}{2}}$ in $H_{2 r, n}$ for every $i \in\left\{0,1, \ldots, \frac{n}{2}-1\right\}$;

- if $k=2 r+1$ and $n$ are odd, then $H_{k, n}$ is obtained from $H_{2 r, n}$ by first linking $v_{0}$ to both $v_{\left\lfloor\frac{n}{2}\right\rfloor}$ and $v_{\left\lceil\frac{n}{2}\right\rceil}$, and then each vertex $v_{i}$ to $v_{i+\left\lceil\frac{n}{2}\right\rceil}$ for every $i \in\left\{1,2, \ldots,\left\lfloor\frac{n}{2}\right\rfloor-1\right\}$;

where the subscripts are taken modulo $n$. Three examples of Harary graphs are given in Figure 1 . When $k$ is odd, the neighbours of a vertex $v_{i}$ of $H_{k, n}$ which are at distance strictly more than $r$ from $v_{i}$ in the underlying $C_{n}$ (there are at most two of them) are called the antipodal neighbours of $v_{i}$. In particular, the vertex $v_{i}$ has two antipodal neighbours if and only if $i=0$, and $k$ and $n$ are both odd. A diagonal edge of $H_{k, n}$ is an edge linking two vertices each of which is an antipodal neighbour of the other one.

If $G$ is a graph with a natural ordering of its vertices (like powers of paths and cycles, or Harary graphs), then, for every vertex $v$ of $G$, we denote by $v^{+}$(resp. $v^{-}$) the neighbour of $v$ succeeding (resp. preceding) $v$ in this ordering. Every power of path $P_{n}^{k}$ with underlying path $P_{n}=v_{0} v_{1} \ldots v_{n-1}$ is considered to be depicted in a "usual" way, i.e. from its leftmost vertex $v_{0}$ to its rightmost vertex $v_{n-1}$. By $u G v$ we refer to the graph $G\left[\left\{u, u^{+},\left(u^{+}\right)^{+}, \ldots, v^{-}, v\right\}\right]$ for every two vertices $u$ and $v$ of $G$. Assuming $P$ is a prescription of $G$, a prescribed block $B$ of $P$ in $G$ is a set $\left\{v_{i_{j_{1}}}, v_{i_{j_{2}}}, \ldots, v_{i_{j_{\ell}}}\right\}$ of consecutive prescribed vertices, i.e. $v_{i_{j_{2}}}=v_{i_{j_{1}}}^{+}, v_{i_{j_{3}}}=v_{i_{j_{2}}}^{+}, \ldots, v_{i_{j_{\ell}}}=v_{i_{j_{\ell}}-1}^{+}$. We say that $B$ is maximal if neither $v_{i_{j_{1}}}^{-}$nor $v_{i_{j_{\ell}}}^{+}$ are prescribed vertices.

One important property of AP graphs is the following.

Observation 2. If a graph $G$ admits a spanning $A P$ (resp. AP+k) subgraph (resp. for some $k \geq 1$ ), then $G$ is $A P($ resp. $A P+k)$.

Recall that a graph is traceable if it admits a Hamiltonian path. Since every path is AP, Observation 2 implies the following result. 
Corollary 3. Every traceable graph is AP.

We now point out the following property of $\mathrm{AP}+k$ graphs, from which we deduce a bound on the size of an optimal $\mathrm{AP}+k$ graph.

Observation 4. Let $k \geq 1$. Every AP+k graph is $(k+1)$-connected. Therefore, an optimal AP+k graph on $n$ vertices has at least $\left\lceil\frac{n(k+1)}{2}\right\rceil$ edges.

Proof: Assume $G$ is a graph with order $n$. If there exist $k$ vertices $v_{1}, v_{2}, \ldots, v_{k}$ such that $G-\left\{v_{1}, v_{2}, \ldots\right.$, $\left.v_{k}\right\}$ is not connected, then the sequence $(1,1, \ldots, 1, n-k)$ with the value 1 appearing $k$ times cannot be realized in $G$ under $\left(v_{1}, v_{2}, \ldots, v_{k}\right)$. Therefore, a necessary condition for $G$ to be $\mathrm{AP}+k$ is to be $(k+1)$-connected. The lower bound then follows.

As mentioned by Corollary 3 , paths are $\mathrm{AP}+0$, while it is easy to check that cycles are $\mathrm{AP}+1$. Baudon et al. generalized these observations to powers of paths and cycles [3].

Theorem 5 ([3]). The graph $P_{n}^{k}$ is $A P+(k-1)$ for every $k \geq 1$ and $n \geq k$. The graph $C_{n}^{k}$ is $A P+(2 k-1)$ for every $k \geq 1$ and $n \geq 2 k$.

Provided that $n \geq 2 k+2$, note that the size of $P_{n}^{k+1}$ is $(k+1)(n-(k+1))+\sum_{i=1}^{k} i$. Then, since $\left|E\left(P_{n}^{k+1}\right)\right|>\left\lceil\frac{n(k+1)}{2}\right\rceil$, an optimal $\mathrm{AP}+k$ graph on $n$ vertices may have less edges than $P_{n}^{k+1}$. On the contrary, every graph $C_{n}^{k}$ is $2 k$-regular and hence is an edge-minimal $2 k$-connected graph. According to Observation 4 it follows that the set of $k^{t h}$ powers of cycles is a set of optimal $\mathrm{AP}+(2 k-1)$ graphs for every $k \geq 1$.

\section{Partitioning powers of paths and cycles under prescriptions}

As pointed out in Theorem 5. recall that $k^{\text {th }}$ powers of paths and cycles are $\mathrm{AP}+(k-1)$ and $\mathrm{AP}+(2 k-1)$, respectively. This result is tight according to Observation 4 , in the sense that we cannot always prescribe more vertices while partitioning these graphs. In this section, we exhibit situations under which these graphs can be partitioned under more prescriptions than indicated by their connectivity.

The following first result asserts that $k^{t h}$ powers of paths can be partitioned under $k$-prescriptions when either the first or the last vertex is prescribed.

Lemma 6 ([3]). Let $P=\left(v_{i_{1}}, v_{i_{2}}, \ldots, v_{i_{k}}\right)$ be a $k$-prescription of $P_{n}^{k}$ with $k \geq 1, n \geq k$ and $0 \leq i_{1}<$ $i_{2}<\ldots<i_{k} \leq n-1$. If $i_{1}=0$ or $i_{k}=n-1$, then every sequence $\tau=\left(n_{1}, n_{2}, \ldots, n_{p}\right)$ admissible for $P_{n}^{k}$ with $p \geq k$ elements is realizable in $P_{n}^{k}$ under $P$.

In the next result, we prove that $k^{t h}$ powers of paths are also partitionable under $k$-prescriptions when the prescribed vertices do not form a prescribed block with size $k$.

Lemma 7. Let $P=\left(v_{i_{1}}, v_{i_{2}}, \ldots, v_{i_{k}}\right)$ be a k-prescription of $P_{n}^{k}$ with $k \geq 1, n \geq k$ and $0 \leq i_{1}<$ $i_{2}<\ldots<i_{k} \leq n-1$. If the prescribed vertices do not form a prescribed block with size $k$, then every sequence $\tau=\left(n_{1}, n_{2}, \ldots, n_{p}\right)$ admissible for $P_{n}^{k}$ with $p \geq k$ elements is realizable in $P_{n}^{k}$ under $P$.

Proof: Let $G=P_{n}^{k}$ for given values of $k \geq 1$ and $n \geq k$. If $s=\sum_{j=k+1}^{p} n_{j} \leq i_{1}$, then a realization of $\tau$ in $G$ under $P$ is $\left(V_{1}, V_{2}, \ldots, V_{p}\right)$ where $\left(V_{k+1}, V_{k+2}, \ldots, V_{p}\right)$ is a realization of $\left(n_{k+1}, n_{k+2}, \ldots, n_{p}\right)$ in the traceable graph $G\left[\left\{v_{0}, v_{1}, \ldots, v_{s-1}\right\}\right]$, and $\left(V_{1}, V_{2}, \ldots, V_{k}\right)$ is a realization of $\left(n_{1}, n_{2}, \ldots, n_{k}\right)$ in $G-\left\{v_{0}, v_{1}, \ldots, v_{s-1}\right\}$ under $P$ which exists according to Theorem 1 
Suppose now that $s>i_{1}$. On the one hand, if $n_{1}>i_{1}$, then a realization of $\tau$ in $G$ under $P$ is $\left(V_{1}^{\prime} \cup\right.$ $\left.V_{1}^{\prime \prime}, V_{2}, V_{3}, \ldots, V_{p}\right)$, where $V_{1}^{\prime}=\left\{v_{0}, v_{1}, \ldots, v_{i_{1}-1}\right\}$ and $\left(V_{1}^{\prime \prime}, V_{2}, V_{3}, \ldots, V_{p}\right)$ is a realization of $\left(n_{1}-\right.$ $\left.i_{1}, n_{2}, \ldots, n_{p}\right)$ in $G-V_{1}^{\prime}$ under $P$ obtained via Lemma 6 . On the other hand, if $n_{1} \leq i_{1}$, then let $V_{1}$ be a subset of $\left\{v_{0}, v_{1}, \ldots, v_{i_{1}}\right\}$ obtained as follows. First, we set $V_{1}=\left\{v_{i_{1}}\right\}$ and we then repeatedly add to $V_{1}$ the vertex located at distance 2 on the left of the last vertex added to $V_{1}$ as long as $\left|V_{1}\right|<n_{1}$ and $v_{0}$ is not reached. If there is no vertex at distance 2 on the left of the last vertex added to $V_{1}$ (but $V_{1}$ needs additional vertices), then we add to $V_{1}$ every remaining vertex from $\left\{v_{0}, v_{1}, \ldots, v_{i_{1}-1}\right\}-V_{1}$ from left to right until $V_{1}$ has size $n_{1}$. Let $X=\left\{v_{0}, v_{1}, \ldots, v_{i_{1}-1}\right\}-V_{1}$. Notice that, at the end of the procedure, $G\left[V_{1}\right]$ is connected, $G[X]$ is traceable, and $v_{i_{1}-1} \in X$. Now, if there exists $r \in\{k+1, k+2, \ldots, p\}$ such that $\sum_{j=k+1}^{r} n_{j}=|X|$, then a realization of $\tau$ in $G$ under $P$ is $\left(V_{1}, V_{2}, \ldots, V_{p}\right)$ where $\left(V_{k+1}, V_{k+2}, \ldots, V_{r}\right)$ is a realization of $\left(n_{k+1}, n_{k+2}, \ldots, n_{r}\right)$ in $G[X]$ and $\left(V_{2}, V_{3}, \ldots, V_{k}, V_{r+1}, V_{r+2}, \ldots, V_{p}\right)$ is a realization of $\left(n_{2}, n_{3}, \ldots, n_{k}, n_{r+1}, n_{r+2}, \ldots, n_{p}\right)$ in $G-\left\{v_{0}, v_{1}, \ldots, v_{i_{1}}\right\}$ under $\left\{v_{i_{2}}, v_{i_{3}} \ldots, v_{i_{k}}\right\}$ obtained using Theorem 5

If such a value of $r$ does not exist, then let $r$ be such that $\sum_{j=k+1}^{r-1} n_{j}<|X|$ and $\sum_{j=k+1}^{r} n_{j}>|X|$. Let further $n_{r}^{\prime}=|X|-\sum_{j=k+1}^{r-1} n_{j}, n_{r}^{\prime \prime}=n_{r}-n_{r}^{\prime}$, and $v_{a} \notin P$ be the nearest neighbour of $v_{i_{1}-1}$ located on the right of $v_{i_{1}}$. Such a vertex necessarily exists since the opposite assumption would imply that our $k$ prescribed vertices are located consecutively along $G$. Moreover, either $v_{a}$ or $v_{i_{2}}$ is the first vertex of $G-\left\{v_{0}, v_{1}, \ldots, v_{i_{1}}\right\}$. We then obtain a realization $\left(V_{1}, V_{2}, \ldots, V_{r-1}, V_{r}^{\prime} \cup V_{r}^{\prime \prime}, V_{r+1}, V_{r+2}, \ldots, V_{p}\right)$ of $\tau$ in $G$ under $P$, where $\left(V_{r}^{\prime}, V_{k+1}, V_{k+2}, \ldots, V_{r-1}\right)$ is a realization of $\left(n_{r}^{\prime}, n_{k+1}, n_{k+2}, \ldots, n_{r-1}\right)$ in $G[X] \operatorname{under}\left(v_{i_{1}-1}\right)$, and $\left(V_{2}, V_{3}, \ldots, V_{k}, V_{r}^{\prime \prime}, V_{r+1}, V_{r+2}, \ldots, V_{p}\right)$ is a realization of $\left(n_{2}, n_{3}, \ldots, n_{k}, n_{r}^{\prime \prime}\right.$, $\left.n_{r+1}, n_{r+2}, \ldots, n_{p}\right)$ in $G\left[\left\{v_{i_{1}+1}, v_{i_{1}+2}, \ldots, v_{n-1}\right\}\right]$ under $\left(v_{i_{2}}, v_{i_{3}}, \ldots, v_{i_{k}}, v_{a}\right)$. These two realizations exist according to Lemma 6 .

We now strengthen Lemma 6 by showing that $k^{\text {th }}$ powers of paths are partitionable under $(k+1)$ prescriptions when their endvertices are prescribed.

Lemma 8. Let $P=\left(v_{i_{1}}, v_{i_{2}}, \ldots, v_{i_{k+1}}\right)$ be a $(k+1)$-prescription of $P_{n}^{k}$ with $k \geq 1, n \geq k$ and $0 \leq i_{1}<i_{2}<\ldots<i_{k+1} \leq n-1$. If $i_{1}=0$ and $i_{k+1}=n-1$, then every sequence $\tau=\left(n_{1}, n_{2}, \ldots, n_{p}\right)$ admissible for $P_{n}^{k}$ with $p \geq k+1$ elements is realizable in $P_{n}^{k}$ under $P$.

Proof: We prove this claim by induction on $k$. For $k=1$, the result is obvious. We thus now suppose that $k \geq 2$ and that the claim holds for every $k^{\prime}<k$. Let $G=P_{n}^{k}$. If $n_{1} \leq i_{2}$, then a realization of $\tau$ in $G$ under $P$ is $\left(V_{1}, V_{2}, \ldots, V_{p}\right)$ where $V_{1}=\left\{v_{0}, v_{1}, \ldots, v_{n_{1}-1}\right\}$ and $\left(V_{2}, V_{3}, \ldots, V_{p}\right)$ is a realization of $\left(n_{2}, n_{3}, \ldots, n_{p}\right)$ in $G-V_{1}$ under $\left(v_{i_{2}}, v_{i_{3}}, \ldots, v_{i_{k+1}}\right)$. This realization necessarily exists according to Lemma 6 since $v_{i_{k+1}}$ is the last vertex of $G-V_{1}$.

Suppose now that $n_{1}>i_{2}$. Observe that $\{0,1, \ldots, k-1\}-\bigcup_{j=2}^{k}\left\{i_{j} \bmod k\right\}$ is not empty, so let us denote by $r$ one of its elements. The subset $V_{1}$ of the realization is constructed as follows. It first contains all the vertices between $v_{0}$ and $v_{i_{2}-1}$, i.e. $\left\{v_{0}, v_{1}, \ldots, v_{i_{2}-1}\right\} \subseteq V_{1}$. We then add the vertex $v_{a}$ to $V_{1}$, where $a \in\left\{i_{2}+1, i_{2}+2, \ldots, i_{2}+k-1\right\}$ is such that $a \equiv r \bmod k$. Finally, as long as $\left|V_{1}\right|<n_{1}$, we repeatedly add to $V_{1}$ the vertex at distance $k$ on the right from the last vertex added to $V_{1}$, unless it is equal to $v_{n-1}$, i.e. $v_{a+k}$, then $v_{a+2 k}$, and so on. According to our choice of $r$, these vertices are not prescribed ones and, at any moment of the procedure, the subgraph $G-V_{1}$ is spanned by the $(k-1)^{t h}$ power of a path, and the subgraph $G\left[V_{1}\right]$ is connected.

On the one hand, if $\left|V_{1}\right|=n_{1}$ holds after the procedure, then $\left(V_{1}, V_{2}, \ldots, V_{p}\right)$ is a realization of $\tau$ under $P$, where $\left(V_{2}, V_{3}, \ldots, V_{p}\right)$ is a realization of $\left(n_{2}, n_{3}, \ldots, n_{p}\right)$ in $G-V_{1}$ under the prescription 
$\left(v_{i_{2}}, v_{i_{3}}, \ldots, v_{i_{k+1}}\right)$ which necessarily exists by the induction hypothesis since $v_{i_{2}}$ and $v_{i_{k+1}}$ are the endvertices of $G-V_{1}$.

On the other hand, if $\left|V_{1}\right|<n_{1}$ holds once the procedure is achieved, then each vertex from $V(G)-V_{1}$ has a neighbour in $V_{1}$. Hence, we can obtain a realization $\left(V_{1} \cup V_{1}^{\prime}, V_{2}, V_{3}, \ldots, V_{p}\right)$ of $\tau$ in $G$ under $P$, where $\left(V_{2}, V_{3}, \ldots, V_{p}, V_{1}^{\prime}\right)$ is a realization of $\left(n_{2}, n_{3}, \ldots, n_{p}, n_{1}-\left|V_{1}\right|\right)$ in $G-V_{1}$ under the prescription $\left(v_{i_{2}}, v_{i_{3}}, \ldots, v_{i_{k+1}}\right)$. Once again, such a realization necessarily exists according to the induction hypothesis.

We now prove an analogous result concerning cycles to the power of at least 2. Let $G=C_{n}^{k}$ for some $k \geq 2$ and $n \geq 2 k$, the sequence $\tau=\left(n_{0}, n_{1}, \ldots, n_{p-1}\right)$ be admissible for $G$, and $P=$ $\left(v_{i_{0}}, v_{i_{1}}, \ldots, v_{i_{2 k-1}}\right)$ be a $2 k$-prescription of $G$, with $p \geq 2 k$ and $0 \leq i_{0}<i_{1}<\ldots<i_{2 k-1} \leq n-1$. For every $j \in\{0,1, \ldots, 2 k-1\}$, we denote by $D_{j}$ the set $\left\{v_{i_{j-1}}^{+},\left(v_{i_{j-1}}^{+}\right)^{+}, \ldots, v_{i_{j}}^{-}, v_{i_{j}}\right\}$ containing the consecutive vertices of $G$ lying between $v_{i_{j-1}}$ and $v_{i_{j}}$, including $v_{i_{j}}$. The size of every $D_{j}$ is denoted $d_{j}$. In particular, we have $\sum_{j=0}^{2 k-1} d_{j}=n$.

Lemma 9. Let $P=\left(v_{i_{0}}, v_{i_{1}}, \ldots, v_{i_{2 k-1}}\right)$ be a $2 k$-prescription of $C_{n}^{k}$ with $k \geq 2, n \geq 2 k$ and $0 \leq i_{0}<$ $i_{1}<\ldots<i_{2 k-1} \leq n-1$. If the prescribed vertices are not organized into two maximal prescribed blocks with size $k$, then every sequence $\tau=\left(n_{0}, n_{1}, \ldots, n_{p-1}\right)$ admissible for $C_{n}^{k}$ with $p \geq 2 k$ elements is realizable in $C_{n}^{k}$ under $P$.

Proof: Let $k \geq 2$ be fixed, and $G=C_{n}^{k}$ for some value of $n \geq 2 k$. We prove that every partition $\tau=\left(n_{0}, n_{1}, \ldots, n_{p-1}\right)$ of $n$ with $p \geq 2 k$ elements is realizable in $G$ under every $2 k$-prescription $P=$ $\left(v_{i_{0}}, v_{i_{1}}, \ldots, v_{i_{2 k-1}}\right)$ with $0 \leq i_{0}<i_{1}<\ldots<i_{2 k-1} \leq n-1$ when the prescribed vertices do not form two maximal prescribed blocks with size $k$. For every $j \in\{0,1, \ldots, 2 k-1\}$, let $q_{j}=\sum_{\ell=j}^{j+k-1} d_{\ell}$ and $s_{j}=\sum_{\ell=j}^{j+k-1} n_{\ell}$, where the indices are counted modulo $2 k$. In other words, the value $q_{j}$ is the order of the graph $v_{i_{j-1}}^{+} G v_{i_{j+k-1}}=G\left[\left\{i_{j-1}^{+},\left(i_{j-1}^{+}\right)^{+}, \ldots, i_{j+k-1}\right\}\right]$ including the $k$ prescribed vertices $v_{i_{j}}$, $v_{i_{j+1}}, \ldots, v_{i_{j+k-1}}$, and $s_{j}$ is the amount of vertices needed by the subgraphs containing these prescribed vertices in a realization of $\tau$ in $G$ under $P$. Note that there necessarily exists a $j$ such that $s_{j} \leq q_{j}$ since having $\sum_{j=0}^{2 k-1} s_{j} \geq \sum_{j=0}^{2 k-1}\left(q_{j}+1\right)$ implies $k \sum_{\ell=0}^{2 k-1} n_{\ell} \geq k \sum_{\ell=0}^{2 k-1} d_{\ell}+2 k$, which is impossible since $n=\sum_{\ell=0}^{2 k-1} d_{\ell}$ and $n \geq \sum_{\ell=0}^{2 k-1} n_{\ell}$. To prove the claim, we distinguish several cases depending on the relationship between $s_{j}$ 's and $q_{j}$ 's.

Case 1. $s_{j}=q_{j}$ for some $j \in\{0,1, \ldots, 2 k-1\}$.

In this situation, a realization of $\tau$ in $G$ under $P$ is deduced as follows. Assume $j=0$ without loss of generality, and set $G_{0}=G\left[\bigcup_{\ell=0}^{k-1} D_{\ell}\right]$. Note that $G_{0}$ is the $k^{t h}$ power of a path. If $\sum_{\ell=0}^{k-1} d_{\ell} \geq k+1$, then $G_{0}$ is $k$-connected and thus admits a realization $\left(V_{0}, V_{1}, \ldots, V_{k-1}\right)$ of $\left(n_{0}, n_{1}, \ldots, n_{k-1}\right)$ under $\left(v_{i_{0}}, v_{i_{1}}, \ldots, v_{i_{k-1}}\right)$ according to Theorem 1 . Otherwise, if $\sum_{\ell=0}^{k-1} d_{\ell}=$ $k$, then $\left(V_{0}, V_{1}, \ldots, V_{k-1}\right)=\left(\left\{v_{i_{0}}\right\},\left\{v_{i_{1}}\right\}, \ldots,\left\{v_{i_{k-1}}\right\}\right)$ is a realization of $\left(n_{0}, n_{1}, \ldots, n_{k-1}\right)=$ $(1,1, \ldots, 1)$ in $G_{0}$ under $\left(v_{i_{0}}, v_{i_{1}}, \ldots, v_{i_{k-1}}\right)$. On the other hand, the graph $G-\bigcup_{\ell=0}^{k-1} D_{\ell}$ is the $k^{\text {th }}$ power of a path whose last vertex is $v_{i_{2 k-1}}$. Therefore, there exists a realization $\left(V_{k}, V_{k+1}, \ldots, V_{p-1}\right)$ of $\left(n_{k}, n_{k+1}, \ldots, n_{p-1}\right)$ under $\left(v_{i_{k}}, v_{i_{k+1}}, \ldots, v_{i_{2 k-1}}\right)$ in this graph by Lemma 6 . The partition $\left(V_{0}, V_{1}, \ldots, V_{p-1}\right)$ is then a realization of $\tau$ in $G$ under $P$. 
Case 2. We are not in Case 1 and $s_{j}>q_{j}$ for some $j \in\{0,1, \ldots, 2 k-1\}$.

In particular, there exists a value of $j$ for which $s_{j}>q_{j}$ and $s_{j+1}<q_{j+1}$. Suppose $j=0$ without loss of generality.

Case 2.1. There exists a set $X=\left\{v_{i_{2 k-1}}^{+},\left(v_{i_{2 k-1}}^{+}\right)^{+}, \ldots, v_{a}\right\}$ with $a \in\left\{i_{k-1}+1, i_{k-1}+2, \ldots, i_{k}-\right.$ 1) such that $|X|=s_{0}$.

A realization of $\tau$ in $G$ under $P$ can be obtained as follows. Firstly, let $\left(V_{0}, V_{1}, \ldots, V_{k-1}\right)$ be a realization of $\left(n_{0}, n_{1}, \ldots, n_{k-1}\right)$ in $G[X]$ under $\left(v_{i_{0}}, v_{i_{1}}, \ldots, v_{i_{k-1}}\right)$. Such a realization exists by Theorem 1 since $G[X]$ is the $k^{t h}$ power of a path. Secondly, let $\left(V_{k}, V_{k+1}, \ldots, V_{p-1}\right)$ be a realization of $\left(n_{k}, n_{k+1}, \ldots, n_{p-1}\right)$ in $G-X$ under $\left(v_{i_{k}}, v_{i_{k+1}}, \ldots, v_{i_{2 k-1}}\right)$ which necessarily exists according to Lemma 6 since $v_{i_{2 k-1}}$ is the last vertex of $G-X$. The partition $\left(V_{0}, V_{1}, \ldots, V_{p-1}\right)$ is then a realization of $\tau$ in $G$ under $P$.

Case 2.2. Such a set $X$ does not exist.

In such a situation, we have $s_{0}>q_{0}+d_{k}-1$, i.e. $\sum_{\ell=0}^{k-1} n_{\ell}>\sum_{\ell=0}^{k} d_{\ell}-1$. Besides, since $n_{\ell}$ 's and $d_{\ell}$ 's are strictly greater than 0 , we get $\sum_{\ell=0}^{k} n_{\ell} \geq 1+\sum_{\ell=1}^{k} d_{\ell}$. Since $s_{1}<q_{1}$, i.e. $\sum_{\ell=1}^{k} n_{\ell}<\sum_{\ell=1}^{k} d_{\ell}$, it follows that there exists a $n_{0}^{\prime}$ such that $1 \leq n_{0}^{\prime} \leq n_{0}$ and $n_{0}^{\prime}+\sum_{\ell=1}^{k} n_{\ell}=1+\sum_{\ell=1}^{k} d_{\ell}=\left|\left\{v_{i_{0}}, v_{i_{0}}^{+}, \ldots, v_{i_{k}}\right\}\right|$. A realization of $\tau$ in $G$ under $P$ is then obtained as follows. On the one hand, let $\left(V_{0}^{\prime}, V_{1}, V_{2}, \ldots, V_{k}\right)$ be a realization of $\left(n_{0}^{\prime}, n_{1}, n_{2}, \ldots, n_{k}\right)$ in $G\left[\left\{v_{i_{0}}, v_{i_{0}}^{+}, \ldots, v_{i_{k}}\right\}\right]$ under $\left(v_{i_{0}}, v_{i_{1}}, \ldots, v_{i_{k}}\right)$, which exists according to Lemma 8 since $v_{i_{0}}$ and $v_{i_{k}}$ are the endvertices of $G\left[\left\{v_{i_{0}}, v_{i_{0}}^{+}, \ldots, v_{i_{k}}\right\}\right]$. On the other hand, let $n_{0}^{\prime \prime}=\left(n_{0}-n_{0}^{\prime}\right)+1$ (note that $n_{0}^{\prime \prime} \geq 1$ ), and let $\left(V_{0}^{\prime \prime}, V_{k+1}, V_{k+2}, \ldots, V_{p-1}\right)$ be a realization of $\left(n_{0}^{\prime \prime}, n_{k+1}, n_{k+2}, \ldots, n_{p-1}\right)$ in $G\left[\left\{v_{i_{k}}^{+},\left(v_{i_{k}}^{+}\right)^{+}, \ldots, v_{i_{0}}\right\}\right]$ under $\left(v_{i_{0}}, v_{i_{k+1}}, v_{i_{k+2}}, \ldots\right.$, $\left.v_{i_{2 k-1}}\right)$, which exists according to Lemma 6 since $G\left[\left\{v_{i_{k}}^{+},\left(v_{i_{k}}^{+}\right)^{+}, \ldots, v_{i_{0}}\right\}\right]$ is the $k^{t h}$ power of a path with last vertex $v_{i_{0}}$, and $k$ prescribed vertices are specified. The partition $\left(V_{0}^{\prime} \cup\right.$ $\left.V_{0}^{\prime \prime}, V_{1}, V_{2}, \ldots, V_{p-1}\right)$ is then a realization of $\tau$ in $G$ under $P$ since $G\left[V_{0}^{\prime}\right]$ and $G\left[V_{0}^{\prime \prime}\right]$ are connected and both contain the vertex $v_{i_{0}}$ (which is actually the only vertex appearing in both these subgraphs).

Case 3. $s_{j}<q_{j}$ for every $j \in\{0,1, \ldots, 2 k-1\}$.

We distinguish two subcases.

Case 3.1. There are two consecutive prescribed vertices. Assume $v_{i_{0}}=v_{i_{2 k-1}}^{+}$without loss of generality, with $i_{0}=0$ and $i_{2 k-1}=n-1$.

Case 3.1.1. There exists $r \in\{2 k, 2 k+1, \ldots, p-1\}$ such that $s_{0}+\sum_{\ell=2 k}^{r} n_{\ell}=q_{0}$. In this situation, we can deduce a realization of $\tau$ in $G$ under $P$ as follows. Firstly, let

$$
\left(V_{0}, V_{1}, \ldots, V_{k-1}, V_{2 k}, V_{2 k+1}, \ldots, V_{r}\right)
$$

be a realization of

$$
\left(n_{0}, n_{1}, \ldots, n_{k-1}, n_{2 k}, n_{2 k-1}, \ldots, n_{r}\right)
$$

in $G\left[\bigcup_{\ell=0}^{k-1} D_{\ell}\right]$ under $\left(v_{i_{0}}, v_{i_{1}}, \ldots, v_{i_{k-1}}\right)$ which exists according to Lemma 6 since $v_{i_{0}}$ is the first vertex of $G\left[\bigcup_{\ell=0}^{k-1} D_{\ell}\right]$, this graph being the $k^{t h}$ power of some path. Secondly, let

$$
\left(V_{k}, V_{k+1}, \ldots, V_{2 k-1}, V_{r+1}, V_{r+2}, \ldots, V_{p-1}\right)
$$


be a realization of

$$
\left(n_{k}, n_{k+1}, \ldots, n_{2 k-1}, n_{r+1}, n_{r+2}, \ldots, n_{p-1}\right)
$$

in $G-\bigcup_{\ell=0}^{k-1} D_{\ell}$ under $\left(v_{i_{k}}, v_{i_{k+1}}, \ldots, v_{i_{2 k-1}}\right)$ which exists for the same reason as previously since $v_{i_{2 k-1}}$ is the last vertex of $G-\bigcup_{\ell=0}^{k-1} D_{\ell}$. The partition $\left(V_{0}, V_{1}, \ldots, V_{p-1}\right)$ is then a realization of $\tau$ in $G$ under $P$.

Case 3.1.2. Such $r$ does not exist.

Let $r \in\{2 k, 2 k+1, \ldots, p-1\}$ be the value for which we have $s_{0}+\sum_{\ell=2 k}^{r-1} n_{\ell}<q_{0}$ and $s_{0}+\sum_{\ell=2 k}^{r} n_{\ell}>q_{0}$. Such a value exists since $s_{0}<q_{0}$ and $s_{k}<q_{k}$. So let further $n_{r}^{\prime}=q_{0}-\left(s_{0}+\sum_{\ell=2 k}^{r-1} n_{\ell}\right)$ and $n_{r}^{\prime \prime}=n_{r}-n_{r}^{\prime}$. Denote by $v_{a}$ the last non-prescribed vertex of $G\left[\bigcup_{\ell=0}^{k-1} D_{\ell}\right]$, and by $v_{b}$ the first non-prescribed vertex of $G-\bigcup_{\ell=0}^{k-1} D_{\ell}$.

Case 3.1.2.1. The vertices $v_{a}$ and $v_{b}$ are adjacent in $G$.

Let $v_{b}=v_{i_{q}}^{+}$for some $q \in\{k+1, k+2, \ldots, 2 k-2\}$. Then we obtain a realization of $\tau$ in $G$ under $P$ as follows. Firstly, let

$$
\left(V_{0}, V_{1}, \ldots, V_{k-1}, V_{r}^{\prime}, V_{2 k}, V_{2 k+1}, \ldots, V_{r-1}\right)
$$

be a realization of $\left(n_{0}, n_{1}, \ldots, n_{k-1}, n_{r}^{\prime}, n_{2 k}, n_{2 k+1}, \ldots, n_{r-1}\right)$ in $G\left[\bigcup_{\ell=0}^{k-1} D_{\ell}\right]$ under $\left(v_{i_{0}}, v_{i_{1}}, \ldots, v_{i_{k-1}}, v_{a}\right)$, which exists by Lemma 8 since $G\left[\bigcup_{\ell=0}^{k-1} D_{\ell}\right]$ is the $k^{\text {th }}$ power of a path whose endvertices are $v_{i_{0}}$ and $v_{i_{k-1}}$. Secondly, let

$$
\left(V_{k}, V_{k+1}, \ldots, V_{q}, V_{r}^{\prime \prime}, V_{q+1}, V_{q+2}, \ldots, V_{2 k-1}, V_{r+1}, V_{r+2}, \ldots, V_{p-1}\right)
$$

be a realization of

$$
\left(n_{k}, n_{k+1}, \ldots, n_{q}, n_{r}^{\prime \prime}, n_{q+1}, n_{q+2}, \ldots, n_{2 k-1}, n_{r+1}, n_{r+2}, \ldots, n_{p-1}\right)
$$

in $G-\bigcup_{\ell=0}^{k-1} D_{\ell}$ under $\left(v_{i_{k}}, v_{i_{k+1}}, \ldots, v_{i_{q}}, v_{b}, v_{i_{q+1}}, v_{i_{q+2}}, \ldots, v_{i_{2 k-1}}\right)$.

This realization exists according to Lemma 8 since $G-\bigcup_{\ell=0}^{k-1} D_{\ell}$ is the $k^{t h}$ power of a path, either $v_{b}$ or $v_{i_{k}}$ is the first vertex of $G-\bigcup_{\ell=0}^{k-1} D_{\ell}$, and $v_{i_{2 k-1}}$ is the last vertex of $G-\bigcup_{\ell=0}^{k-1} D_{\ell}$. It follows that

$$
\left(V_{0}, V_{1}, \ldots, V_{r-1}, V_{r}^{\prime} \cup V_{r}^{\prime \prime}, V_{r+1}, V_{r+2}, \ldots, V_{p-1}\right)
$$

is a realization of $\tau$ in $G$ under $P$ since $G\left[V_{r}^{\prime} \cup V_{r}^{\prime \prime}\right]$ is connected thanks to the edge $v_{a} v_{b}$.

Case 3.1.2.2. The vertices $v_{a}$ and $v_{b}$ are not adjacent in $G$.

In this situation, either $v_{i_{k-1}}$ or $v_{i_{k}}$ belongs to a prescribed block with size at least $k$. Then one can relabel the prescribed vertices so that $v_{i_{0}}$ and $v_{i_{2 k-1}}$ correspond to two consecutive prescribed vertices from this prescribed block, and use the procedures from Case 3.1. Since $s_{j}<q_{j}$ for every $j \in\{0,1, \ldots, 2 k-1\}$, note that this time the two vertices $v_{a}$ and $v_{b}$ (if these vertices are needed) have to be adjacent since otherwise it would mean that the prescribed vertices form another prescribed block with size at least $k$, implying that there are two prescribed blocks with size $k$, contradicting the assumption of the lemma. 
Case 3.2. There are no two consecutive prescribed vertices.

Case 3.2.1. There exists a set $X$ of consecutive vertices of $G$ such that $X \cap P=\left\{v_{i_{j}}, v_{i_{j+1}}\right.$, $\left.\ldots, v_{i_{j+k-1}}\right\}$ and $|X|=s_{j}$ for some $j \in\{0,1, \ldots, 2 k-1\}$.

In this situation, we obtain a realization of $\tau$ in $G$ under $P$ as follows. Assume $j=0$ without loss of generality. Firstly, let $\left(V_{0}, V_{1}, \ldots, V_{k-1}\right)$ be a realization of $\left(n_{i_{0}}, n_{i_{1}}, \ldots\right.$, $\left.n_{i_{k-1}}\right)$ in $G[X]$ under $\left(v_{i_{0}}, v_{i_{1}}, \ldots, v_{i_{k-1}}\right)$, which exists by Theorem 1 since $G[X]$ is the $k^{\text {th }}$ power of some path. Secondly, let $\left(V_{k}, V_{k+1}, \ldots, V_{p-1}\right)$ be a realization of $\left(n_{k}, n_{k+1}, \ldots, n_{p-1}\right)$ in $G-X$ under $\left(v_{i_{k}}, v_{i_{k+1}}, \ldots, v_{i_{2 k-1}}\right)$ obtained thanks to Lemma 7 since $G-X$ is the $k^{t h}$ power of a path and there are no consecutive prescribed vertices. Then $\left(V_{0}, V_{1}, \ldots, V_{p-1}\right)$ is a realization of $\tau$ in $G$ under $P$.

Case 3.2.2. $s_{j}<q_{j}-d_{j}+1$ for every $j \in\{0,1, \ldots, 2 k-1\}$.

Case 3.2.2.1. There are two prescribed vertices $v_{i_{\ell}}$ and $v_{i_{\ell+1}}$ such that $n_{\ell}+n_{\ell+1} \geq$ $d_{\ell+1}+1$.

Assume $\ell=2 k-1$ without loss of generality. Then there exist two sets of consecutive vertices $X=\left\{v_{i_{2 k-1}}, v_{i_{2 k-1}}^{+}, \ldots, v_{a}\right\}$ and $Y=\left\{v_{a}^{+},\left(v_{a}^{+}\right)^{+}, \ldots, v_{i_{0}}\right\}$, with $a \in\left\{i_{2 k-1}, i_{2 k-1}+1 \bmod n, \ldots, i_{0}-1 \bmod n\right\},|X| \leq n_{2 k-1}$ and $|Y| \leq n_{0} . \mathrm{A}$ realization of $\tau$ in $G$ under $P$ can be then obtained as in Case 3.1 by doing as if $v_{i_{2 k-1}}$ and $v_{i_{0}}$ were consecutive prescribed vertices (this is straightforward due to the notation we have adopted herein), but requesting $v_{i_{2 k-1}}$ and $v_{i_{0}}$ to belong to subgraphs with order $n_{2 k-1}-|X|+1$ and $n_{0}-|Y|+1$, respectively. Recall that we are under the assumption that there are no two consecutive prescribed vertices. For the resulting parts $V_{2 k-1}^{\prime}$ and $V_{0}^{\prime}$, the graphs $G\left[V_{2 k-1}^{\prime} \cup X\right]$ and $G\left[V_{0}^{\prime} \cup Y\right]$ are connected, and have order $n_{2 k-1}$ and $n_{0}$, respectively.

Case 3.2.2.2. $n_{j}+n_{j+1}<d_{j+1}+1$ for every $j \in\{0,1, \ldots, 2 k-1\}$.

In particular, $n_{0}+n_{1}<d_{1}+1=\left|\left\{v_{i_{0}}, v_{i_{0}}^{+}, \ldots, v_{i_{1}}\right\}\right|$. We cannot have both $n_{0} \geq\left\lceil\frac{d_{1}+1}{2}\right\rceil$ and $n_{1} \geq\left\lceil\frac{d_{1}+1}{2}\right\rceil$, since otherwise we would get $n_{0}+n_{1} \geq d_{1}+1$, a contradiction. Let us thus suppose that $n_{0}<\left\lceil\frac{d_{1}+1}{2}\right\rceil$ without loss of generality. Then note that the graph induced by $V_{0}=\left\{v_{i_{0}}, v_{i_{0}+2}, v_{i_{0}+4}, \ldots, v_{i_{0}+2\left(n_{0}-1\right)}\right\}$ has order $n_{0}$ and contains $v_{i_{0}}$, and the graph $G\left[\left\{v_{i_{2 k-1}}, v_{i_{2 k-1}}^{+}, \ldots, v_{i_{1}}\right\}-V_{0}\right]$ is traceable with endvertices $v_{i_{2 k-1}}$ and $v_{i_{1}}$. Let $t_{1}=\left|\left\{v_{i_{1}}, v_{i_{1}}^{+}, \ldots, v_{i_{k}}\right\}\right|-\sum_{\ell=1}^{k} n_{\ell}$ and $t_{2}=\left|\left\{v_{i_{2 k-1}}^{+},\left(v_{i_{2 k-1}}^{+}\right)^{+}, \ldots, v_{i_{1}}^{-}\right\}\right|-n_{0}$. From $\tau$, we define three sequences $\tau_{1}, \tau_{2}$ and $\tau_{3}$.

First, let $\tau_{1}=\left(n_{1}, n_{2}, \ldots, n_{k}, n_{2 k}, n_{2 k+1}, \ldots, n_{r_{1}-1}\right)$, where $r_{1}$ is the unique index in $\{2 k, 2 k+1, \ldots, p-1\}$ such that $\sum_{\ell=2 k}^{r_{1}-1} n_{\ell} \leq t_{1}$ and $\sum_{\ell=2 k}^{r_{1}} n_{\ell}>t_{1}$. Now, if $t_{1}-\sum_{\ell=2 k}^{r_{1}-1} n_{\ell}>0$, then add $n_{r_{1}}^{\prime}=t_{1}-\sum_{\ell=2 k}^{r_{1}-1} n_{\ell}$ as the $(k+1)^{t h}$ element of $\tau_{1}$. Note that the elements of $\tau_{1}$ sum up to $\left|\left\{v_{i_{1}}, v_{i_{1}}^{+}, \ldots, v_{i_{k}}\right\}\right|$.

Let $n_{r_{1}}^{\prime \prime}=n_{r_{1}}-n_{r_{1}}^{\prime}$. If $n_{r_{1}}^{\prime \prime} \geq t_{2}$, then let $\tau_{2}=\left(t_{2}\right)$, and set $r_{2}=r_{1}$ and $n_{r_{2}}^{\prime \prime}=n_{r_{1}}^{\prime \prime}-t_{2}$. Otherwise, let $r_{2}$ be the index in $\left\{r_{1}+1, r_{1}+2, \ldots, p-1\right\}$ for which $n_{r_{1}}^{\prime \prime}+\sum_{\ell=r_{1}+1}^{r_{2}-1} n_{\ell} \leq$ $t_{2}$ and $n_{r_{1}}^{\prime \prime}+\sum_{\ell=r_{1}+1}^{r_{2}} n_{\ell}>t_{2}$. Now let $\tau_{2}=\left(n_{r_{1}}^{\prime \prime}, n_{r_{1}+1}, n_{r_{1}+2}, \ldots, n_{r_{2}-1}\right)$. Set $n_{r_{2}}^{\prime}=t_{2}-\left(n_{r_{1}}^{\prime \prime}+\sum_{\ell=r_{1}+1}^{r_{2}-1} n_{\ell}\right)$ and $n_{r_{2}}^{\prime \prime}=n_{r_{2}}-n_{r_{2}}^{\prime}$, and add $n_{r_{2}}^{\prime}$ as the second element of $\tau_{2}$ if $n_{r_{2}}^{\prime}>0$. Once $\tau_{2}$ is constructed, note that its elements sum up to $\left|\left\{v_{i_{2 k-1}}^{+},\left(v_{i_{2 k-1}}^{+}\right)^{+}, \ldots, v_{i_{1}}^{-}\right\}\right|-n_{0}$.

Finally, assuming $n_{r_{2}}^{\prime \prime}>0$ (otherwise, remove this element from the sequence), let $\tau_{3}=$ 
$\left(n_{k+1}, n_{k+2}, \ldots, n_{2 k-1}, n_{r_{2}}^{\prime \prime}, n_{r_{2}+1}, n_{r_{2}+2}, \ldots, n_{p-1}\right)$. Note that the elements of $\tau_{3}$ sum up to $\left|\left\{v_{i_{k}}^{+},\left(v_{i_{k}}^{+}\right)^{+}, \ldots, v_{i_{2 k-1}}\right\}\right|$.

Remark that every element of $\tau$ has been associated with one of $\tau_{1}, \tau_{2}$ and $\tau_{3}$, with at most two non-prescribed elements being split so that the $\tau_{i}$ 's sum up exactly to the orders of some subgraphs of $G$. In the case where $\tau$ contains a "big" non-prescribed element, it is even possible that this element was split into three integers among $\tau_{1}, \tau_{2}$ and $\tau_{3}$. To obtain the realization of $\tau$ in $G$ under $P$, we realize $\tau_{1}, \tau_{2}$ and $\tau_{3}$ in vertex-disjoint subgraphs of $G$, and this in such a way that if an original element of $\tau$ was dispatched in several of the $\tau_{i}$ 's, then the resulting connected subgraphs perform a whole connected subgraph when unified.

The three realizations $R_{1}, R_{2}$ and $R_{3}$ are obtained as follows.

- Let $R_{1}$ be a realization of $\tau_{1}$ in $G\left[\left\{v_{i_{1}}, v_{i_{1}}^{+}, \ldots, v_{i_{k}}\right\}\right]$ under $\left(v_{i_{1}}, v_{i_{2}}, \ldots, v_{i_{k}}, v_{i_{1}}^{+}\right)$, which exists according to Lemma 8 since $v_{i_{1}}$ and $v_{i_{k}}$ are the endvertices of $G\left[\left\{v_{i_{1}}\right.\right.$, $\left.\left.v_{i_{1}}^{+}, \ldots, v_{i_{k}}\right\}\right]$ and there are $k+1$ prescribed vertices.

- Let $R_{2}$ be a realization of $\tau_{2}$ in $G\left[\left\{v_{i_{2 k-1}}^{+},\left(v_{i_{2 k-1}}^{+}\right)^{+}, \ldots, v_{i_{1}}^{-}\right\}-V_{0}\right]$, which is traceable by our choice of $V_{0}$. Additionally request the realization to satisfy the prescription $\left(v_{i_{1}}^{-}, v_{i_{2 k-1}}^{+}\right)$when $\tau_{2}$ has at least two elements. Such a requirement is allowed according to Lemma 8 .

- Let $R_{3}$ be a realization of $\tau_{3}$ in $G\left[\left\{v_{i_{k}}^{+},\left(v_{i_{k}}^{+}\right)^{+}, \ldots, v_{i_{2 k-1}}\right\}\right]$ under $\left(v_{i_{k+1}}, v_{i_{k+2}}\right.$ $\left., \ldots, v_{i_{2 k-1}}, v_{i_{2 k-1}}^{-}\right)$. The existence of such a realization follows from Lemma 6 since $G\left[\left\{v_{i_{k}}^{+},\left(v_{i_{k}}^{+}\right)^{+}, \ldots, v_{i_{2 k-1}}\right\}\right]$ is the $k^{t h}$ power of some path whose last vertex is $v_{i_{2 k-1}}$.

The realization of $\tau$ in $G$ under $P$ is obtained by considering $V_{0}$ and the parts from $R_{1}$, $R_{2}$ and $R_{3}$, and unifying those parts whose sizes result from the split of a single element of $\tau$, if necessary. By our choice of the prescribed vertices, these parts have neighbouring vertices (this follows from the facts that $k \geq 2$, and that the prescribed vertices of $P$ are not consecutive), and thus induce connected subgraphs. This completes the proof.

\section{Partitioning Harary graphs under prescriptions}

Harary graphs are trivially AP according to Corollary 3 We here show that we can always prescribe the largest possible number of vertices (with respect to their connectivity) while partitioning these graphs, except for 3-connected Harary graphs. We consider the three kinds of Harary graphs for this purpose.

\subsection{Construction 1: $k$ is even}

The Harary graph $H_{k, n}$ with $k$ even is isomorphic to $C_{n}^{k / 2}$ which is $\mathrm{AP}+(k-1)$ according to Theorem 5 for every $k \geq 2$ and $n \geq 2 k$. We thus derive the following result.

Corollary 10. For every even $k \geq 2$ and $n \geq 2 k$, the Harary graph $H_{k, n}$ is $A P+(k-1)$. 


\subsection{Construction 2: $k$ is odd and $n$ is even}

Let $k \geq 2$ and $n \geq 2 k+1$ be two integers such that $n$ is even. By construction, the Harary graph $H_{2 k+1, n}$ is spanned by $H_{2 k, n}$ and is thus $\mathrm{AP}+(2 k-1)$ according to Corollary 10 However, regarding the connectivity of $H_{2 k+1, n}$, one could wonder whether $H_{2 k+1, n}$ is $\mathrm{AP}+2 k$.

Before proving that $H_{2 k+1, n}$ is indeed $\mathrm{AP}+2 k$, we first introduce the following lemma which deals with the traceability of a graph composed by two linked squares of paths.

Lemma 11. If $G$ is a graph such that $V(G)=V_{1} \cup V_{2}$, the subgraphs $G\left[V_{1}\right]$ and $G\left[V_{2}\right]$ are both spanned by the square of a path, and there exists an edge joining one vertex of $V_{1}$ and one of $V_{2}$, then $G$ is traceable.

Proof: Let $v_{1}, v_{2}, \ldots, v_{\ell}$ and $u_{1}, u_{2}, \ldots, u_{\ell^{\prime}}$ denote the consecutive vertices of $G\left[V_{1}\right]$ and $G\left[V_{2}\right]$, and $v_{a} \in V_{1}$ and $u_{b} \in V_{2}$ be two vertices of $G$ such that $v_{a} u_{b} \in E(G)$. Consider the following subpaths of $G$ :

- $P=v_{1} v_{2} \ldots v_{a-1}$

- $Q=\left\{\begin{array}{l}v_{a+1} v_{a+3} \ldots v_{\ell-1} v_{\ell} v_{\ell-2} v_{\ell-4} \ldots v_{a+2} \text { if } \ell-a \text { is even } \\ v_{a+1} v_{a+3} \ldots v_{\ell} v_{\ell-1} v_{\ell-3} \ldots v_{a+2} \text { otherwise }\end{array}\right.$

- $R=\left\{\begin{array}{l}u_{b+2} u_{b+4} \ldots u_{\ell^{\prime}} u_{\ell^{\prime}-1} u_{\ell^{\prime}-3} \ldots u_{b+1} \text { if } \ell^{\prime}-b \text { is even } \\ u_{b+2} u_{b+4} \ldots u_{\ell^{\prime}-1} u_{\ell^{\prime}} u_{\ell^{\prime}-2} u_{\ell^{\prime}-4} \ldots u_{b+1} \text { otherwise }\end{array}\right.$

- $S=u_{b-1} u_{b-2} \ldots u_{1}$.

It is then easy to check that $P Q v_{a} u_{b} R S$ is a Hamiltonian path of $G$.

We are now ready to prove our main result.

Theorem 12. For every $k \geq 2$ and even $n \geq 2 k+1$, the Harary graph $H_{2 k+1, n}$ is $A P+2 k$.

Proof: Let $k \geq 2$ and even $n \geq 2 k+1$ be fixed, and $G=H_{2 k+1, n}$ be the $(2 k+1)$-connected Harary graph on $n$ vertices. We prove that every sequence $\tau=\left(n_{0}, n_{1}, \ldots, n_{p-1}\right)$, admissible for $G$ with $p \geq 2 k+1$ elements, is realizable in $G$ under every $2 k$-prescription $P=\left(v_{i_{0}}, v_{i_{1}}, \ldots, v_{i_{2 k-1}}\right)$ with $0 \leq i_{0}<i_{1}<\ldots<i_{2 k-1} \leq n-1$. We distinguish two main cases.

Case 1. If the prescribed vertices are not organized into two maximal prescribed blocks with size $k$, then, because $k \geq 2$, we can deduce a realization of $\tau$ in the spanning $C_{n}^{k}$ of $G$ under $P$, thanks to Lemma 9 Such a realization is naturally a realization of $\tau$ in $G$ under $P$.

Case 2. Suppose now that the prescribed vertices form two maximal prescribed blocks $B_{1}$ and $B_{2}$ with size exactly $k$ in $G$. In this situation, note that $G-P$ only remains connected thanks to some diagonal edges. Indeed, assume $B_{1}=\left\{v_{i_{0}}, v_{i_{1}}, \ldots, v_{i_{k-1}}\right\}$ and $B_{2}=\left\{v_{i_{k}}, v_{i_{k+1}}, \ldots, v_{i_{2 k-1}}\right\}$ without loss of generality. Then the antipodal neighbours of $v_{i_{0}}^{-}$and $v_{i_{k-1}}^{+}$cannot both belong to $P$ : since $n \geq 2 k+2$, if this were the case then these two antipodal neighbours would belong to $B_{2}$, and similarly for all antipodal neighbours of $v_{i_{0}}, v_{i_{1}}, \ldots, v_{i_{k-1}}$ (according to our assumptions on the maximal prescribed blocks). We would then get that $B_{2}$ has size at least $k+2$, a contradiction. Let us thus denote by $v_{a}$ and $v_{b}$ two antipodal neighbours of $G$ such that $v_{a}, v_{b} \notin B_{1} \cup B_{2}$. In particular, we may suppose $a \in\left\{i_{k-1}+1, i_{k-1}+2, \ldots, i_{k}-1\right\}$ and $b \in\left\{i_{2 k-1}+1, i_{2 k-1}+2, \ldots, i_{0}-1\right\}$. Let further $a_{1}=a-i_{k-1}-1, a_{2}=i_{k}-a-1, a_{3}=i_{0}-b-1$ and $a_{4}=b-i_{2 k-1}-1$ denote the number of consecutive vertices between $B_{1}, B_{2}$ and the two vertices $v_{a}$ and $v_{b}$ according to the natural ordering of $G$. 
Case 2.1. $\sum_{j=0}^{k-1} n_{j} \leq a_{1}+a_{3}+k$ and $\sum_{j=k}^{2 k-1} n_{j} \leq a_{2}+a_{4}+k$.

In this situation, we can find two subsets $X$ and $Y$ of consecutive vertices of $G$ such that $|X|=\sum_{j=0}^{k-1} n_{j},|Y|=\sum_{j=k}^{2 k-1} n_{j},\left\{v_{i_{0}}, v_{i_{1}}, \ldots, v_{i_{k-1}}\right\} \subseteq X,\left\{v_{i_{k}}, v_{i_{k+1}}, \ldots, v_{i_{2 k-1}}\right\} \subseteq$ $Y$, and $v_{a}, v_{b} \notin X \cup Y$. Since $G[X]$ and $G[Y]$ are both isomorphic to the $k^{\text {th }}$ power of a path, by Theorem 1 we know that we can deduce two realizations $\left(V_{0}, V_{1}, \ldots, V_{k-1}\right)$ and $\left(V_{k}, V_{k+1}, \ldots, V_{2 k-1}\right)$ of $\left(n_{0}, n_{1}, \ldots, n_{k-1}\right)$ and $\left(n_{k}, n_{k+1}, \ldots, n_{2 k-1}\right)$, respectively, in $G[X]$ and $G[Y]$, respectively, under $\left(v_{i_{0}}, v_{i_{1}}, \ldots, v_{i_{k}-1}\right)$ and $\left(v_{i_{k}}, v_{i_{k+1}}, \ldots, v_{i_{2 k-1}}\right)$, respectively. Now, since $k \geq 2$, the graph $G-(X \cup Y)$ is traceable according to Lemma 11 and thus admits a realization $\left(V_{2 k}, V_{2 k+1}, \ldots, V_{p-1}\right)$ of $\left(n_{2 k}, n_{2 k+1}, \ldots, n_{p-1}\right)$. Finally, the partition $\left(V_{0}, V_{1}, \ldots, V_{p-1}\right)$ is a realization of $\tau$ in $G$ under $P$.

Case 2.2. $\sum_{j=0}^{k-1} n_{j}>a_{1}+a_{3}+k$ without loss of generality.

Note that we have $\sum_{j=0}^{2 k-1} n_{j} \geq \min \left\{a_{1}+a_{2}+2 k+1, a_{3}+a_{4}+2 k+1\right\}$, since otherwise

$$
a_{1}+a_{3}+2 k+1 \leq \sum_{j=0}^{k-1} n_{j}+\sum_{j=k}^{2 k-1} n_{j}=\sum_{j=0}^{2 k-1} n_{j}<\frac{1}{2}\left(a_{1}+a_{2}+a_{3}+a_{4}\right)+2 k+1,
$$

which implies $a_{1}+a_{3}<a_{2}+a_{4}$, a contradiction. Then we consider two new cases.

Case 2.2.1. $\sum_{j=0}^{2 k-1} n_{j} \geq a_{1}+a_{2}+2 k+1$.

Under this assumption, we can find two subsets of consecutive vertices $X, Y \subseteq V(G)$ such that $\left\{v_{i_{0}}, v_{i_{1}}, \ldots, v_{i_{k-1}}\right\} \subseteq X,\left\{v_{i_{k}}, v_{i_{k+1}}, \ldots, v_{i_{2 k-1}}\right\} \subseteq Y,|X|=\sum_{j=0}^{k-1} n_{j}$, $|Y|=\sum_{j=k}^{2 k-1} n_{j}$, and the last vertex of $G[X]$ is the vertex preceding the first vertex of $G[Y]$. By Theorem 11, we know that we can deduce realizations $\left(V_{0}, V_{1}, \ldots, V_{k-1}\right)$ and $\left(V_{k}, V_{k+1}, \ldots, V_{2 k-1}\right)$ of $\left(n_{0}, n_{1}, \ldots, n_{k-1}\right)$ and $\left(n_{k}, n_{k+1}, \ldots, n_{2 k-1}\right)$, respectively, in $G[X]$ and $G[Y]$, respectively, under $\left(v_{i_{0}}, v_{i_{1}}, \ldots, v_{i_{k-1}}\right)$ and $\left(v_{i_{k}}, v_{i_{k+1}}, \ldots, v_{i_{2 k-1}}\right)$, respectively. Finally, since the graph $G-(X \cup Y)$ is isomorphic to the $k^{t h}$ power of a path, there exists a realization $\left(V_{2 k}, V_{2 k+1}, \ldots, V_{p}\right)$ of the remaining sequence $\left(n_{2 k}, n_{2 k+1}, \ldots\right.$, $\left.n_{p-1}\right)$ in it. We get that $\left(V_{0}, V_{1}, \ldots, V_{p-1}\right)$ is a realization of $\tau$ in $G$ under $P$.

Case 2.2.2. $\sum_{j=0}^{2 k-1} n_{j} \geq a_{3}+a_{4}+2 k+1$.

In this case, we proceed similarly as in Case 2.2.1, but the last vertex of $G[Y]$ has to be the vertex preceding the first vertex of $G[X]$.

\subsection{Construction 3: $k$ and $n$ are odd}

Since two Harary graphs $H_{2 k+1, n}$ and $H_{2 k+1, n^{\prime}}$, with $k \geq 2$, and $n \geq 2 k+1$ and $n^{\prime} \geq 2 k+1$ being even and odd, respectively, are both spanned by $C_{n}^{k}$, Case 1 from the proof of Theorem 12 also holds directly regarding Harary graphs with odd connectivity and order. Despite $H_{2 k+1, n}$ and $H_{2 k+1, n^{\prime}}$ slightly differ by their diagonal edges, it is easy to realize that if the assumptions of Case $\mathbf{2}$ from the proof of Theorem 12 are fulfilled, that proof can be adapted for considering Harary graphs of odd connectivity and order.

Theorem 13. For every $k \geq 2$ and odd $n \geq 2 k+1$, the Harary graph $H_{2 k+1, n}$ is $A P+2 k$. 


\section{On the existence of optimal AP+2 graphs}

Recall that Theorems 12 and 13 exclude 3-connected Harary graphs, mainly because some of their subgraphs do not satisfy the traceability property exhibited in Lemma 11. Therefore, our proof cannot be used to prove that 3-connected Harary graphs are AP+2.

Besides, it turns out that 3-connected Harary graphs are not all AP+2 anyway. A straight argument for that claim follows from the fact that an unbalanced bipartite graph $G=(A \cup B, E)$, i.e. such that $|A| \neq|B|$, with even order does not admit a perfect matching.

Lemma 14. If a bipartite graph $G=(A \cup B, E)$ has even (resp. odd) order, then, assuming $G$ has enough vertices, the graph $G$ cannot be $A P+k$ for every even (resp. odd) $k \geq 2$ (resp. $k \geq 1$ ).

Proof: We prove the claim for bipartite graphs with even order, but the proof is analogous for bipartite graphs with odd order. Let $k \geq 2$ be even and fixed. For such a value of $k$, we can find two subsets $X \subset A$ and $Y \subset B$ such that $X \cap Y=\emptyset,|X|+|Y|=k$, and $|A-X| \neq|B-Y|$. Let $A^{\prime}=A-X$ and $B^{\prime}=B-X$. Then since $\left|A^{\prime}\right|+\left|B^{\prime}\right|$ is even and $\left|A^{\prime}\right| \neq\left|B^{\prime}\right|$, the graph $G\left[A^{\prime} \cup B^{\prime}\right]$ cannot admit a perfect matching. It follows that the sequence $(1,1, \ldots, 1,2,2, \ldots, 2)$, with the value 1 appearing $k$ times, is not realizable in $G$ under $\left(v_{1}, v_{2}, \ldots, v_{k}\right)$, where $\left\{v_{1}, v_{2}, \ldots, v_{k}\right\}=X \cup Y$.

Corollary 15. For every $n \equiv 2 \bmod 4$, the Harary graph $H_{3, n}$ is not $A P+2$.

Proof: This follows from Lemma 14 since every such Harary graph is a balanced bipartite graph.

In order to prove that there actually exist optimal $\mathrm{AP}+2$ graphs on $n$ vertices and $\left\lceil\frac{3 n}{2}\right\rceil$ edges for every $n \geq 4$, we introduce another class of 3-connected graphs. Let $n \geq 4$. The graph $\operatorname{Pr}_{n}$ is constructed as follows.

- If $n$ is even, then $P r_{n}$ is obtained from the cycle $C_{n}$, whose vertices are successively denoted by $u, w_{1}^{1}, w_{2}^{1}, \ldots, w_{\frac{n-2}{2}}^{1}, v, w_{\frac{n-2}{2}}^{2}, w_{\frac{n-2}{2}-1}^{2} \ldots, w_{1}^{2}$, by adding to it the edge $u v$, and the edge $w_{i}^{1} w_{i}^{2}$ for every $i \in\left\{1,2, \ldots, \frac{n-2}{2}\right\}$.

- If $n$ is odd, then $\operatorname{Pr}_{n}$ is obtained by first removing the edges $w_{1}^{1} w_{1}^{2}$ and $w_{\frac{n-3}{2}}^{1} w_{\frac{n-3}{2}}^{2}$ from $\operatorname{Pr}_{n-1}$, and then adding to it a new vertex $o$ and the edges $o w_{1}^{1}, o w_{1}^{2}, o w_{\frac{n-3}{2}}^{1}$, and $o w_{\frac{n-3}{2}}^{2}$.

Two examples of such graphs are drawn in Figure 2. For every $n \geq 4$, the graph $\operatorname{Pr}_{n}$ is an edgeminimal 3-connected graph since it has size $\left\lceil\frac{3 n}{2}\right\rceil$. To prove that $\operatorname{Pr}$ graphs are $\mathrm{AP}+2$, we consider the following sufficient condition for a graph to be $\mathrm{AP}+2$. Recall that a graph $G$ is Hamiltonian-connected if $G$ admits a Hamiltonian path with endvertices $u$ and $v$ for every two vertices $u$ and $v$ of $G$.

Lemma 16. If a graph $G$ is Hamiltonian-connected, then $G$ is $A P+2$.

Proof: The statement follows from Lemma 8 since every path $P_{n}$ can be partitioned under every 2prescription $(u, v)$ as long as $u$ and $v$ are the endvertices of $P_{n}$.

Before showing that $G=P r_{n}$ is Hamiltonian-connected for every $n \geq 4$, we first introduce some notation. Let $q=\frac{n-2}{2}$ (resp. $q=\frac{n-3}{2}$ ) if $n$ is even (resp. odd). Given two integers $x$ and $y$ in $\{1,2, \ldots, q\}$ (resp. $\{2,3, \ldots, q-1\}$ ) such that $x \leq y$, we denote by $P_{x, y}^{\nearrow}(G)$ and $P_{x, y}^{\searrow}(G)$ the following paths of $G$. 


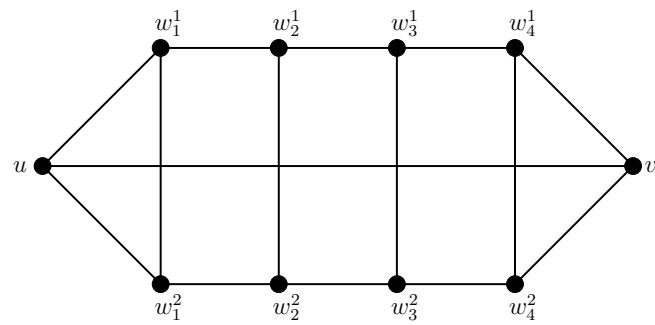

(a) $\operatorname{Pr}_{10}$

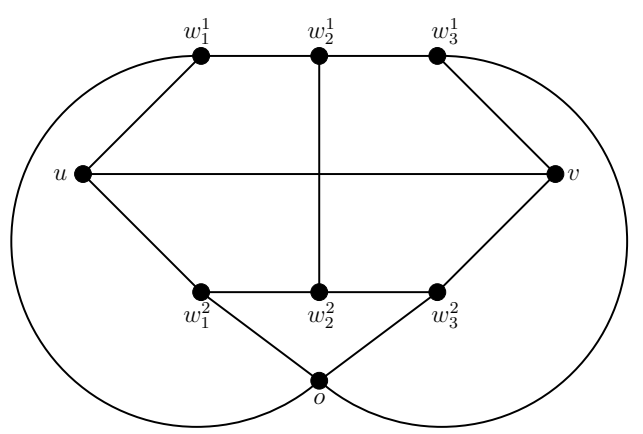

(b) $\operatorname{Pr}_{9}$.

Fig. 2: Two examples of $\operatorname{Pr}$ graphs.

$$
\begin{aligned}
& P_{x, y}^{\nearrow}(G)=\left\{\begin{array}{l}
w_{x}^{2} w_{x}^{1} \text { if } x=y, \\
w_{x}^{2} w_{x}^{1} P_{x+1, y}^{\searrow}(G) \text { otherwise. }
\end{array}\right. \\
& P_{x, y}^{\searrow}(G)=\left\{\begin{array}{l}
w_{x}^{1} w_{x}^{2} \text { if } x=y, \\
w_{x}^{1} w_{x}^{2} P_{x+1, y}^{\nearrow}(G) \text { otherwise. }
\end{array}\right.
\end{aligned}
$$

The paths $P_{x, y}^{\nwarrow}(G)$ and $P_{x, y}^{\swarrow}(G)$ of $G$ are defined analogously from right to left when $x \geq y$. For every $\alpha \in\{1,2\}$, we additionally define $P_{x, y}^{\alpha, \rightarrow}(G)$ (resp. $P_{x, y}^{\alpha, \leftarrow}(G)$ ) for $x<y$ (resp. $x>y$ ) to be the path $w_{x}^{\alpha} w_{x+1}^{\alpha} \ldots w_{y}^{\alpha}$ (resp. $\left.w_{x}^{\alpha} w_{x-1}^{\alpha} \ldots w_{y}^{\alpha}\right)$ of $G$. For convenience, let us assume that $P_{x, y}^{\nearrow}(G)=$ $P_{x, y}^{\searrow}(G)=P_{x, y}^{\alpha, \rightarrow}(G)=\emptyset$ (resp. $P_{x, y}^{\nwarrow}(G)=P_{x, y}^{\swarrow}(G)=P_{x, y}^{\alpha, \leftarrow}(G)=\emptyset$ ) whenever $x$ or $y$ does not belong to the interval above or when $x>y$ (resp. $x<y$ ). According to our terminology, note e.g. that $u P_{1,4}^{1, \rightarrow}\left(\operatorname{Pr}_{10}\right) v P_{4,1}^{2, \leftarrow}\left(\operatorname{Pr}_{10}\right)$ and $u P_{1,4}^{\nearrow}\left(\operatorname{Pr}_{10}\right) v$ are Hamiltonian paths of $\operatorname{Pr}_{10}$.

We are now ready to prove that every $P r_{n}$ graph is Hamiltonian-connected, and thus AP+2 according to Lemma16,

Theorem 17. For every $n \geq 4$, the graph $P r_{n}$ is Hamiltonian-connected.

Proof: Let $G=P r_{n}$, and $q=\frac{n-2}{2}$ if $n$ is even, or $q=\frac{n-3}{2}$ otherwise. Table 1 (resp. Table 2) exhibits, given two distinct vertices $s$ and $t$ of $G$, a Hamiltonian path $P$ of $G$ whose endvertices are $s$ and $t$ when $n$ is even (resp. $n$ is odd). In Table 1 (resp. Table 2), it is assumed that $1 \leq i \leq q$ when $j$ is not defined (resp. $1<i<q$ ), and $0 \leq i<j \leq q$ otherwise (resp. $1<i<j<q$ ). Every Hamiltonian path which does not appear in these two tables can be deduced from another Hamiltonian path using the symmetries of $G$.

Corollary 18. For every $n \geq 4$, the graph $\mathrm{Pr}_{n}$ is $A P+2$. 


\begin{tabular}{|c|c|c|}
\hline$s$ & $t$ & $P$ \\
\hline$u$ & $v$ & $u P_{1, q}^{\nearrow}(G) v$ \\
\hline$u$ & $w_{i}^{1}$ & $\begin{array}{r}u P_{1, i-1}^{\nearrow}(G) w_{i}^{2} P_{i+1, q}^{2, \rightarrow}(G) v P_{q, i}^{1, \leftarrow}(G) \text { if } i-1 \text { is even } \\
u P_{1, i-1}^{\searrow}(G) w_{i}^{2} P_{i+1, q}^{2, \rightarrow}(G) v P_{q, i}^{1, \leftarrow}(G) \text { otherwise }\end{array}$ \\
\hline$w_{i}^{1}$ & $w_{j}^{1}$ & $\begin{array}{l}P_{i, j-1}^{1, \rightarrow}(G) P_{j-1, i}^{2, \leftarrow}(G) P_{i-1,1}^{\nwarrow}(G) u v P_{q, j}^{\nwarrow}(G) \text { if } q-j \text { is even } \\
P_{i, j-1}^{1, \rightarrow}(G) P_{j-1, i}^{2, \leftarrow}(G) P_{i-1,1}^{\nwarrow}(G) u v P_{q, j}^{\swarrow}(G) \text { otherwise }\end{array}$ \\
\hline$w_{i}^{1}$ & $w_{j}^{2}$ & $\begin{array}{l}P_{i, j-1}^{1, \rightarrow}(G) P_{j-1, i}^{2, \leftarrow}(G) P_{i-1,1}^{\nwarrow}(G) u v P_{q, j}^{\swarrow}(G) \text { if } q-j \text { is even } \\
\quad P_{i, j-1}^{1, \rightarrow}(G) P_{j-1, i}^{2, \leftarrow}(G) P_{i-1,1}^{\nwarrow}(G) u v P_{q, j}^{\nwarrow}(G) \text { otherwise }\end{array}$ \\
\hline
\end{tabular}

Tab. 1: Proof that $\operatorname{Pr}_{n}$ is Hamiltonian-connected for every even $n \geq 4$.

\begin{tabular}{|c|c|c|}
\hline$s$ & $t$ & $P$ \\
\hline$O$ & $u$ & $o P_{1, q}^{1, \rightarrow}(G) v P_{q, 1}^{2, \leftarrow}(G) u$ \\
\hline$O$ & $w_{1}^{1}$ & $\begin{array}{l}o w_{q}^{1} v w_{q}^{2} P_{q-1,2}^{\nwarrow} w_{1}^{2} u w_{1}^{1} \text { if } q \text { is even } \\
o w_{q}^{2} v w_{q}^{1} P_{q-1,2}^{\swarrow} w_{1}^{2} u w_{1}^{1} \text { otherwise }\end{array}$ \\
\hline$O$ & $w_{i}^{1}$ & $\begin{array}{l}o w_{1}^{1} u w_{1}^{2} P_{2, i-1}^{\nearrow}(G) w_{i}^{2} P_{i+1, q}^{2, \rightarrow}(G) v P_{q, i}^{1, \leftarrow}(G) \text { if } i \text { is even } \\
\quad o w_{1}^{2} u w_{1}^{1} P_{2, i-1}^{\searrow}(G) w_{i}^{2} P_{i+1, q}^{2, \rightarrow}(G) v P_{q, i}^{1, \leftarrow}(G) \text { otherwise }\end{array}$ \\
\hline$u$ & $v$ & $u P_{1, q}^{2, \rightarrow}(G) o P_{1, q}^{1, \rightarrow}(G) v$ \\
\hline$u$ & $w_{1}^{1}$ & $u v P_{q, 1}^{2, \leftarrow}(G) o P_{q, 1}^{1, \leftarrow}(G)$ \\
\hline$u$ & $w_{q}^{1}$ & $u v P_{q, 1}^{2, \leftarrow}(G) o P_{1, q}^{1, \rightarrow}(G)$ \\
\hline$u$ & $w_{i}^{1}$ & $\begin{array}{l}u P_{1, i-1}^{1, \rightarrow}(G) P_{i-1,1}^{2, \leftarrow}(G) o w_{q}^{2} v w_{q}^{1} P_{q-1, i}^{\swarrow}(G) \text { if } q-i \text { is even } \\
\quad u P_{1, i-1}^{1, \rightarrow}(G) P_{i-1,1}^{2, \leftarrow}(G) o w_{q}^{1} v w_{q}^{2} P_{q-1, i}^{\nwarrow}(G) \text { otherwise }\end{array}$ \\
\hline$w_{1}^{1}$ & $w_{1}^{2}$ & $w_{1}^{1} u v P_{q, 2}^{1, \leftarrow}(G) P_{2, q}^{2, \rightarrow}(G) o w_{1}^{2}$ \\
\hline$w_{1}^{1}$ & $w_{q}^{1}$ & $P_{1, q-1}^{1, \rightarrow}(G) P_{q-1,1}^{2, \leftarrow}(G) u v w_{q}^{2} o w_{q}^{1}$ \\
\hline$w_{1}^{1}$ & $w_{q}^{2}$ & $w_{1}^{1} o w_{1}^{2} u v P_{q, 2}^{1, \leftarrow}(G) P_{2, q}^{2, \rightarrow}(G)$ \\
\hline$w_{i}^{1}$ & $w_{j}^{1}$ & $\begin{array}{c}P_{i, j-1}^{1, \rightarrow}(G) P_{j-1, i}^{2, \leftarrow}(G) P_{i-1,2}^{\nwarrow}(G) w_{1}^{2} u w_{1}^{1} o w_{q}^{2} v w_{q}^{1} P_{q-1, j}^{\swarrow}(G) \text { if } i \text { and } q-j \text { are even } \\
P_{i, j-1}^{1, \rightarrow}(G) P_{j-1, i}^{2, \leftarrow}(G) P_{i-1,2}^{\nwarrow}(G) w_{1}^{1} u w_{1}^{2} o w_{q}^{2} v w_{q}^{1} P_{q-1, j}^{\swarrow}(G) \text { if } i \text { is odd and } q-j \text { is even } \\
P_{i, j-1}^{1, \rightarrow}(G) P_{j-1, i}^{2, \leftarrow}(G) P_{i-1,2}^{\nwarrow}(G) w_{1}^{2} u w_{1}^{1} o w_{q}^{1} v w_{q}^{2} P_{q-1, j}^{\nwarrow}(G) \text { if } i \text { is even and } q-j \text { is odd } \\
\quad P_{i, j-1}^{1, \rightarrow}(G) P_{j-1, i}^{2, \leftarrow}(G) P_{i-1,2}^{\nwarrow}(G) w_{1}^{1} u w_{1}^{2} o w_{q}^{1} v w_{q}^{2} P_{q-1, j}^{\nwarrow}(G) \text { if } i \text { and } q-j \text { are odd }\end{array}$ \\
\hline$w_{i}^{1}$ & $w_{j}^{2}$ & $\begin{array}{c}P_{i, j-1}^{1, \rightarrow}(G) P_{j-1, i}^{2, \leftarrow}(G) P_{i-1,2}^{\nwarrow}(G) w_{1}^{2} u w_{1}^{1} o w_{q}^{1} v w_{q}^{2} P_{q-1, j}^{\nwarrow}(G) \text { if } i \text { and } q-j \text { are even } \\
P_{i, j-1}^{1, \rightarrow}(G) P_{j-1, i}^{2, \leftarrow}(G) P_{i-1,2}^{\nwarrow}(G) w_{1}^{1} u w_{1}^{2} o w_{q}^{1} v w_{q}^{2} P_{q-1, j}^{\nwarrow}(G) \text { if } i \text { is odd and } q-j \text { is even } \\
P_{i, j-1}^{1, \rightarrow}(G) P_{j-1, i}^{2, \leftarrow}(G) P_{i-1,2}^{\nwarrow}(G) w_{1}^{2} u w_{1}^{1} o w_{q}^{2} v w_{q}^{1} P_{q-1, j}^{\swarrow}(G) \text { if } i \text { is even and } q-j \text { is odd } \\
\quad P_{i, j-1}^{1, \rightarrow}(G) P_{j-1, i}^{2, \leftarrow}(G) P_{i-1,2}^{\nwarrow}(G) w_{1}^{1} u w_{1}^{2} o w_{q}^{2} v w_{q}^{1} P_{q-1, j}^{\swarrow}(G) \text { if } i \text { and } n-j \text { are odd }\end{array}$ \\
\hline
\end{tabular}

Tab. 2: Proof that $\operatorname{Pr}_{n}$ is Hamiltonian-connected for every odd $n \geq 5$. 


\section{Conclusion}

We summarize Corollaries 10 and 18 and Theorems 12 and 13 in this concluding theorem.

Theorem 19. For every $k \geq 1$ and $n \geq k$, there exists an optimal $A P+k$ graph on $n$ vertices and $\left\lceil\frac{n(k+1)}{2}\right\rceil$ edges.

This result does not tell much about the number of optimal $\mathrm{AP}+k$ graphs on $n$ vertices for some fixed values of $k$ and $n$. However, this number is upper bounded by the number of edge-minimal $(k+1)$ connected graphs with order $n$ according to Observation 4

\section{Acknowledgements}

The authors would like to thank the anonymous referees for their careful reading and their numerous valuable comments.

\section{References}

[1] D. Barth, O. Baudon, and J. Puech. Decomposable trees: a polynomial algorithm for tripodes. Discrete Appl. Math., 119(3):205-216, July 2002.

[2] D. Barth and H. Fournier. A degree bound on decomposable trees. Discrete Math., 306(5):469-477, 2006.

[3] O. Baudon, J. Bensmail, J. Przybyło, and M. Woźniak. Partitioning powers of traceable or hamiltonian graphs. Theor. Comput. Sci., 520:133-137, 2014.

[4] J. Bensmail. On the complexity of partitioning a graph into a few connected subgraphs. To appear in J. Comb. Optim. DOI 10.1007/s10878-013-9642-8.

[5] E. Győri. On division of graphs to connected subgraphs. In Combinatorics, pages 485-494, Colloq. Math. Soc. János Bolyai 18, 1978.

[6] M. Horňák and M. Woźniak. Arbitrarily vertex decomposable trees are of maximum degree at most six. Opuscula Math., 23:49-62, 2003.

[7] L. Lovász. A homology theory for spanning trees of a graph. Acta Math. Acad. Sci. Hung., 30(34):241-251, 1977.

[8] A. Marczyk. An Ore-type condition for arbitrarily vertex decomposable graphs. Discrete Math., 309(11):3588-3594, 2009. 